\title{
Preparation and characterization of Poly(vinyl alcohol)/graphene nanofibers synthesized by electrospinning
}

Farshad Barzegar', Abdulhakeem Bello', Mopeli Fabiane', Saleh Khamlich', Damilola Momodu', Fatemeh Taghizadeh ${ }^{1}$, Julien Dangbegnon ${ }^{1}$, Ncholu Manyala ${ }^{1}$

${ }^{1}$ Department of Physics, SARChl Chair in Carbon Technology and Materials, Institute of Applied Materials, University of Pretoria, Pretoria, South Africa

\begin{abstract}
We report on the synthesis and characterization of electrospun polyvinyl alcohol (PVA)/graphene nanofibers. The samples produced were characterized by Raman spectroscopy for structural and defect density analysis, scanning electron microscopy (SEM) for morphological analysis, and thermogravimetric (TGA) for thermal analysis. SEM measurements show uniform hollow PVA fibers formation and excellent graphene dispersion within the fibers, while TGA measurements show the improved thermal stability of PVA in the presence of graphene. The synthesized polymer reinforced nanofibers have potential to serve in many different applications such as thermal management, supercapacitor electrodes and biomedical materials for drug delivery.
\end{abstract}

Keywords: electrospinning; nanofiber; nanotechnology; PVA/graphene nanofiber

\section{Introduction}

Fibers have become attractive due to their high length to diameter ratio at the nanoscale and have several potential industrial and commercial applications [1]. The properties of the nanofibers which makes them commercially important includes small diameter, large surface area and small pore size; which are ideal industrial requirements for filtration, catalysis and adsorption $[2,3]$. There are several techniques for production of nanofibers such as drawing, template synthesis, phase separation, self-assembly and electrospinning $[1,4]$. Amongst this techniques electrospinning is versatile for production of continuous fibers from micro scale to nanometer range [5] by the application of electrostatic forces to a jetting polymer solution $[6,7]$. Mechanical reinforcement of fibers 
by the incorporation of nanofillers has been shown as a method for enhancement of the fibers' properties for a number of particular applications. Materials mostly used as nanofillers include zero dimensional (OD) nanoparticles [8], One dimensional (1D) nanotubes [9-11] and two dimensional (2D) layered materials $[8,12,13]$. The incorporation of these materials into the matrix generally improves the mechanical, electrical, thermal, and optical properties of the fiber. Examples of 2D-layered nanofillers used include graphene and graphene oxide (GO) $[14,15]$ owing to their relatively high mechanical strength, and thermal and electrical conductivities [16] which make them promising candidates as nanofillers for enhancement of the fiber properties. It has been shown that the addition of these materials into polymer matrix can greatly improve the mechanical $[17,18]$, electrical [19] and thermal stability [20] of the polymer fibers.

In most cases, electrospinning polymer matrix $\left(\mathrm{TiO}_{2}-\mathrm{PVP}\right)$ gives rise to hollow fibers which are usually stabilized with sol-gel as reported by Jesse et. al [21]. This sol-gel serves as nano fillers and hence an increase in the morphological homogeneity of the resulting nanofibers. Recently graphitic material such as graphene derived from expanded graphite (EG) have been used as fillers by centrifuge and ultrasonication in polyvinylpyrrolidone (PVP)/PVA polymer matrix, but PVP leads to fragile fiber when electrospun [22]. This may be due to the fact that electrospinning of polymer/graphene composite matrix solution requires a homogeneous well dispersed graphene sheets in the polymer matrix solution [2] which is not easily achievable using EG and could also be due to the aggregation or agglomeration of the graphene sheets due to the dispersion forces (van der Waals and Casimir interactions) [23]. In this study, we explore the prospects of uniform dispersion of graphene foam and graphene derived from expanded graphite in hollow PVA polymer matrix nanofibers achieved by just optimizing growth parameters to enhance and/or modify their properties via electrospinning. 


\section{Experimental}

Nickel foam (Alantum Innovation, Munich, Germany), Expandable graphite (grade ES 250 B5 from Qingdao Kropfmuehi Graphite), Polyvinyl alcohol (PVA) ( $M_{w} 89,000$ $98,000 \mathrm{~g} \mathrm{~mol}^{-1}$ Sigma Aldrich) were used as received for the preparation of materials to be used in the synthesis of fibers.

\subsection{Materials Preparation}

Graphene foam (GF) was synthesized on nickel foam template with $420 \mathrm{~g} / \mathrm{m}^{2}$ in area density and $1.6 \mathrm{~mm}$ in thickness, using CVD technique as previously reported [24,25].. Briefly the nickel foam was annealed at $800{ }^{\circ} \mathrm{C}$ in the presence of $\mathrm{H}_{2}$ :Ar flow ratio of 10:300 sccm for 20 minutes to remove impurities, before the introduction of the $\mathrm{CH}_{4}$ gas at $1000{ }^{\circ} \mathrm{C}$. The flow rates of the gases $\left(\mathrm{CH}_{4}: \mathrm{H}_{2}: \mathrm{Ar}\right)$ were 10:10:300 sccm, respectively. After 15 minutes of deposition, the sample was rapidly cooled by pushing the quartz tube to a lower temperature region. After growth, the nickel template was removed by chemical means as follows: polymethylmethacrylate (PMMA) was drop-coated on the sample and baked at $180{ }^{\circ} \mathrm{C}$ for 30 minutes to provide mechanical support for the GF during etching of the nickel. The samples were then placed in $3 \mathrm{M} \mathrm{HCl}$ solution at $80^{\circ} \mathrm{C}$, left overnight to ensure complete removal of the nickel template. The resulting GF sample was placed in acetone at $50{ }^{\circ} \mathrm{C}$ for 30 minutes to remove the PMMA and subsequently placed in isopropanol at $80^{\circ} \mathrm{C}$ for 1 hour to remove the acetone and finally rinsed in deionized water to remove the isopropanol. A subsequent annealing was performed at $400{ }^{\circ} \mathrm{C}$ in argon. After annealing, the sample was again rinsed with deionized water and dried at $50{ }^{\circ} \mathrm{C}$ in an electric oven. The $\mathrm{EG}$ samples were synthesized by exfoliation of expandable graphite in a quartz boat using a microwave oven at $300 \mathrm{~W}$ irradiated for 5 minutes.

The composites solutions were prepared by ultrasonication (Eumax sonicator (ud100sh)) for both GF and EG in $100 \mathrm{ml}$ of $10 \mathrm{wt} . \%$ PVA solution for 5 hours at $40{ }^{\circ} \mathrm{C}$ with varying mass ratios of the starting materials ranging from 20 to $80 \mathrm{mg}$ in order to obtain a good homogeneous dispersion of graphene sheets. The solution was then centrifuged at $5000 \mathrm{rpm}$ for 3 minutes to remove aggregates from the GF and EG. The ultrasonication step for EG in the PVA solution may lead to exfoliation of the graphite to 
graphene sheets [2]. The composite solution was also centrifuged to remove the nonexfoliated graphite aggregates.

\section{$2.2 \quad$ Electrospinning}

The homogeneous dispersed solutions were electrospun using a standard NaBond electrospinning machine (NaBond Technologies SN -50F6). Before production of the nanofibers the various parameters such as the flow rates, the voltage and the speed were optimized to get the best fibers without beads and these values were further used to perform the subsequent experiments. The solution was loaded into a hypodermic syringe $(10 \mathrm{ml})$ and a flexible tube was used to connect the syringe to the blunt needles (Gauge 6). A syringe pump was used to control the flow rate of the solution which was varied between 3 and $7 \mathrm{ml} / \mathrm{h}$. The applied voltage between the needle and the collector was adjusted between 10 and $30 \mathrm{kV}$ and the fibers were collected on a glass (18 $\times 18$ $\mathrm{mm}$ ) substrate mounted on the rotating collector by conductive tape with speed varying between 500 to $1000 \mathrm{rpm}$. The distance between the needle and the collector was varied between 10 and $14 \mathrm{~cm}$ and the growth time between 30 to 180 seconds. Figure 1 show the schematic diagram of the fiber growth process.
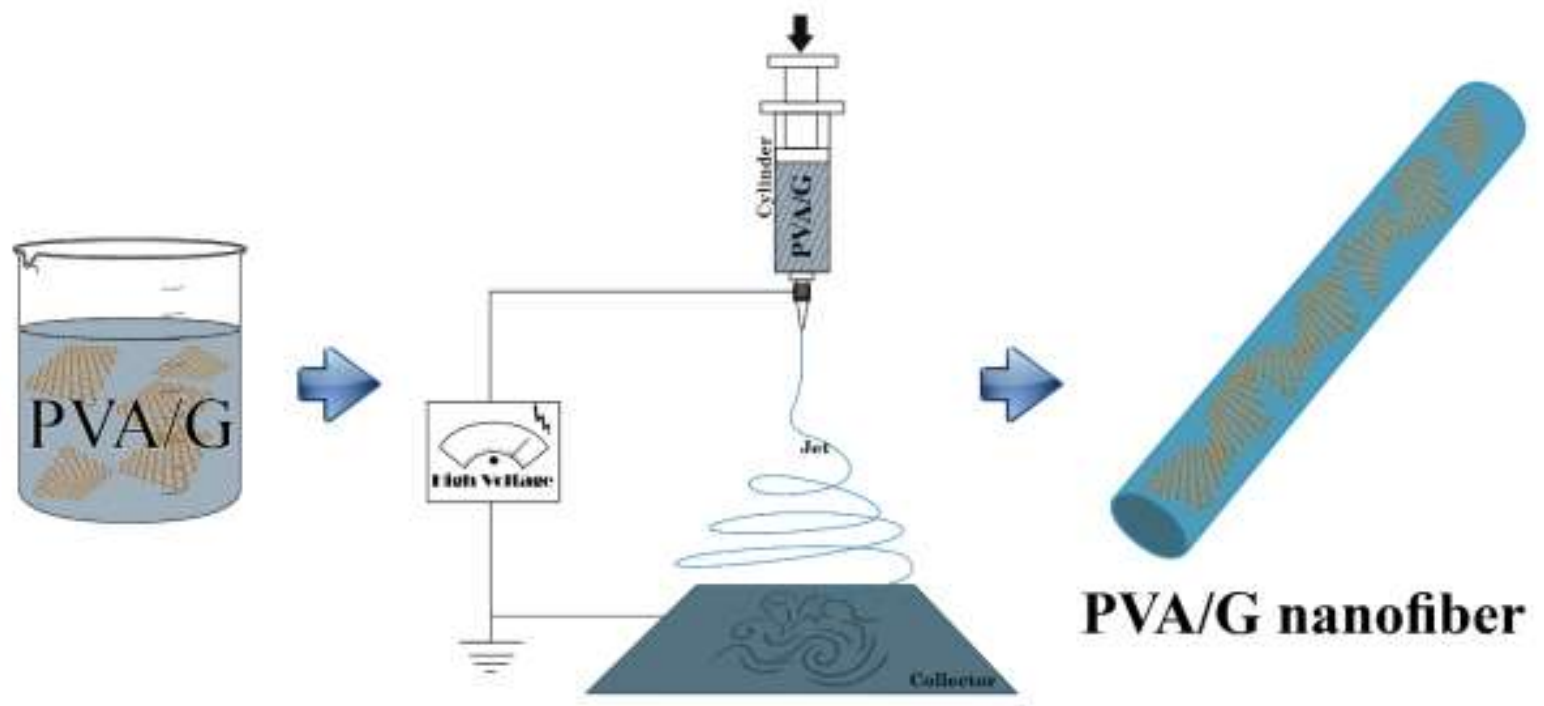

Figure 1 Schematic illustration of the fabrication of graphene/polymer nanofiber composite by electrospinning 


\subsection{Characterization}

Raman spectroscopic analysis of the graphene and composite materials were performed using a T64000 micro-Raman spectrometer from HORIBA Scientific, Jobin Yvon Technology equipped with a triple monochromator system to eliminate contributions from the Rayleigh line. All the samples were analyzed with a $514 \mathrm{~nm}$ argon excitation laser with a power of $12 \mathrm{~mW}$ at laser exit to avoid thermal effects. The surface morphology of all samples was investigated using a Zeiss Ultra Plus 55 field emission scanning electron microscope (FE-SEM). Thermogravimetric Analysis (TGA) was carried out using TA Instruments Q600 Simultaneous DSC/TG which measures the weight change in a material as a function of temperature or time under a controlled atmosphere. TGA samples were heated from room temperature to $1000{ }^{\circ} \mathrm{C}$ at a rate of $10^{\circ} \mathrm{C} \cdot \mathrm{min}^{-1}$ in air.

\subsection{Results and discussion}

\subsection{Raman analysis}

a)

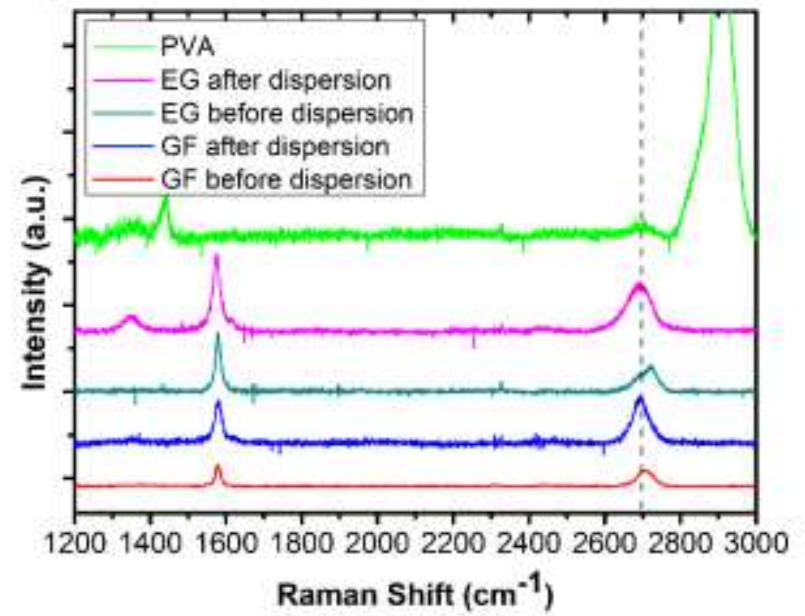

b)

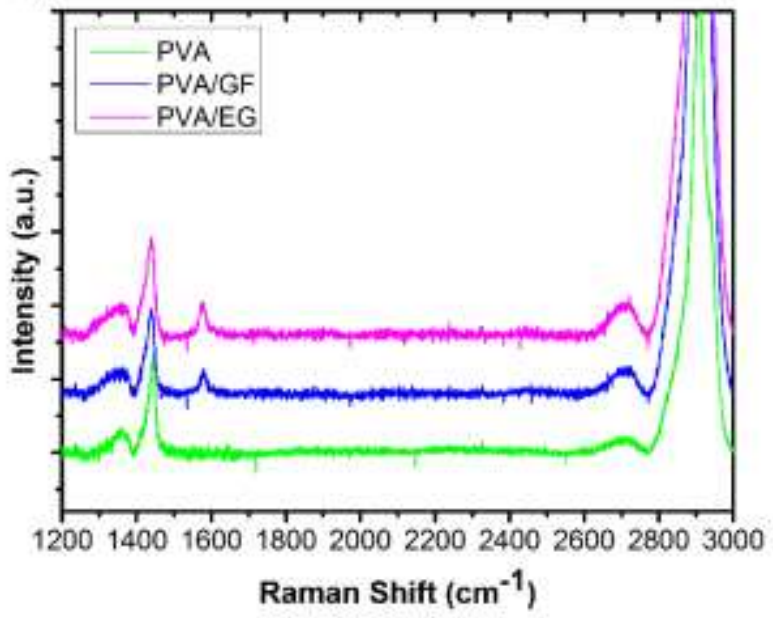

Figure 2 Raman spectra of (a) PVA, GF (before and after being dispersed) and EG (before and after being dispersed) (b) PVA, PVA/GF and PVA/EG

Raman spectroscopy is an important tool for characterization of carbon materials. Figure 2 (a) and (b) show Raman spectra of PVA, GF and EG before and after 
dispersed in water respectively. The dispersed GF and EG spectra show prominent peaks at around $1580 \mathrm{~cm}^{-1}$ (G-Peak) and $2700 \mathrm{~cm}^{-1}$ (2D-Peak). G-Peak have been assigned to graphitic carbons with $\mathrm{sp}^{2}$ hybridization $[26,27]$. In particular, the 2D peak of the EG dispersed in water and ultrasonicated matches the wavenumber of the shoulder of the 2D mode in the EG before sonication. This shift of the 2D to lower wavelength is generally observed for graphene [26]. This evidences the exfoliation of graphite to graphene after dispersion in solvent by ultrasonication. In addition, in the case of disordered sample EG after dispersion, the peak at around $1350 \mathrm{~cm}^{-1}$ (D-Peak) is observed which corresponds to defect in the graphene sample. The full width at half maximum (FWHM) of the 2D peak for dispersed GF and EG are 54 and 76, respectively while the $I_{2 D}: I_{G}$ intensity ratios are $\sim 1$ and 0.78 for dispersed GF and $E G$, respectively. These values show that the produced samples are few layer graphene sheets [28]. The Raman spectrum of PVA shows peaks at $1360 \mathrm{~cm}^{-1}$ and $1440 \mathrm{~cm}^{-1}$ which correspond to the $\mathrm{C}-\mathrm{H}$ and $\mathrm{O}-\mathrm{H}$ bending. Additional peaks at about $2700 \mathrm{~cm}^{-1}$ and $2900 \mathrm{~cm}^{-1}$ are attributed to $\mathrm{C}-\mathrm{H}$ stretching in PVA [29].

Figure 2 (b) Compares Raman spectra of PVA/graphene composites samples that were drop-coated on glass substrate and dried for 15 minutes at $60^{\circ} \mathrm{C}$ in oven. All prominent peaks from graphene and PVA as indicated in figures 2 (a) above are still observed with exception of the reduction in intensity of $2 \mathrm{D}$ peak of graphene when dispersed in PVA solution. This reduction of the $2 \mathrm{D}$ peak is due to the fact that the peak is more susceptible to defects which could be due to interaction of the graphene flakes and PVA molecules indicating a well and homogeneous dispersion. This is also supported by prominent appearance of $D$ peak in all solutions even for PVA/GF which were not observed in GF spectra in figure 2 (a).

\subsection{Nanofibers Morphology}

The morphologies of the fibers, can be controlled by different parameters such as solution concentration, flow rate, applied voltage, duration of electrospinning and distance between needle tips to collector $[3,30,31]$. Prior to the electrospinning the PVA/graphene solutions, the system has been optimized with pure PVA solution to 
produce smooth and thin PVA fibers without beads and drops. The best optimized conditions were used for the growth of fibers using the composite solutions. The optimized parameters are as follows: PVA concentration $=10 \mathrm{wt}$. $\%$, flow rate $=3 \mathrm{ml} / \mathrm{h}$, applied voltage $=30 \mathrm{kV}$, growth time $=60 \mathrm{~s}$ and tip-collector distance $=12 \mathrm{~cm}$. These parameters yielded fibers with diameters of $\sim 238-302 \mathrm{~nm}$, measured from the SEM micrographs. These fibers have hollow structure as shown in figure 3 and indicated by

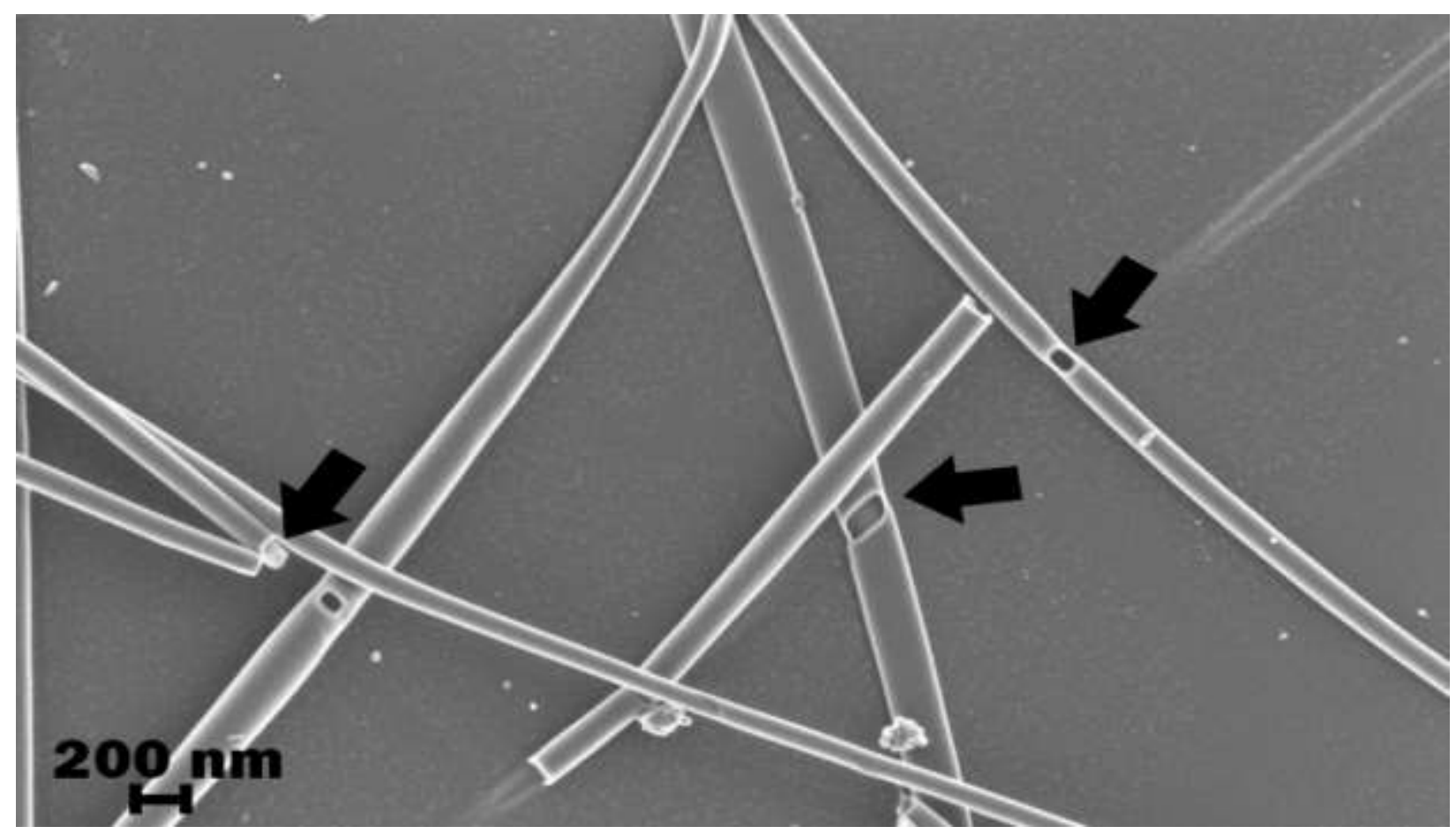

Figure 3 SEM micrographs of PVA hollow nanofibers

the arrows. This hollow structure shows that the fibers can be filled with some nanofillers such as graphene to enhance properties of the fibers. This structure is in line with hollow nanofibers produced using different methods such as self-assembly and template materials [21,32]. It is worthwhile mentioning that the exact formation mechanism of hollow fibers in only one solvent has not been fully elucidated, however we think that the formation of hollow fibers during the process could be due to polar behavior of the solvent used here which was water. When the electric field applied from very high voltage of $30 \mathrm{keV}$ between the needle and the collector, water molecules will be polarized by this high electric field and consequently aligning and forming the core fluid with the PVA forming the shield during the electrospinning, resulting in hollow 
structure formation. This is sustained by the high volume of water used in this experiment since only $10 \mathrm{wt}$. \% of PVA is present in the electrospun aqueous solution.

The SEM results show that before adding graphene the average diameter of fibers were in the range $\sim 238$ - $302 \mathrm{~nm}$ and after adding graphene the average diameter decreased to the range130 - $230 \mathrm{~nm}$. This shows that addition of graphene to the solution decreased the diameter of fibers. This could be due to the fact that graphene enhanced some properties of the starting solution such as electrical conductivity which has a great influence on the diameters of the fibers. The more conducting the solution is the better chance of getting thinner fibers. Thus incorporation of graphene into PVA solution enhanced the conductivity of the solution to be electrospun and because of this improved conductivity the resulting fibers became thinner compared to fibers produced with non-conducting material such as PVA $[1,3,33,34]$.
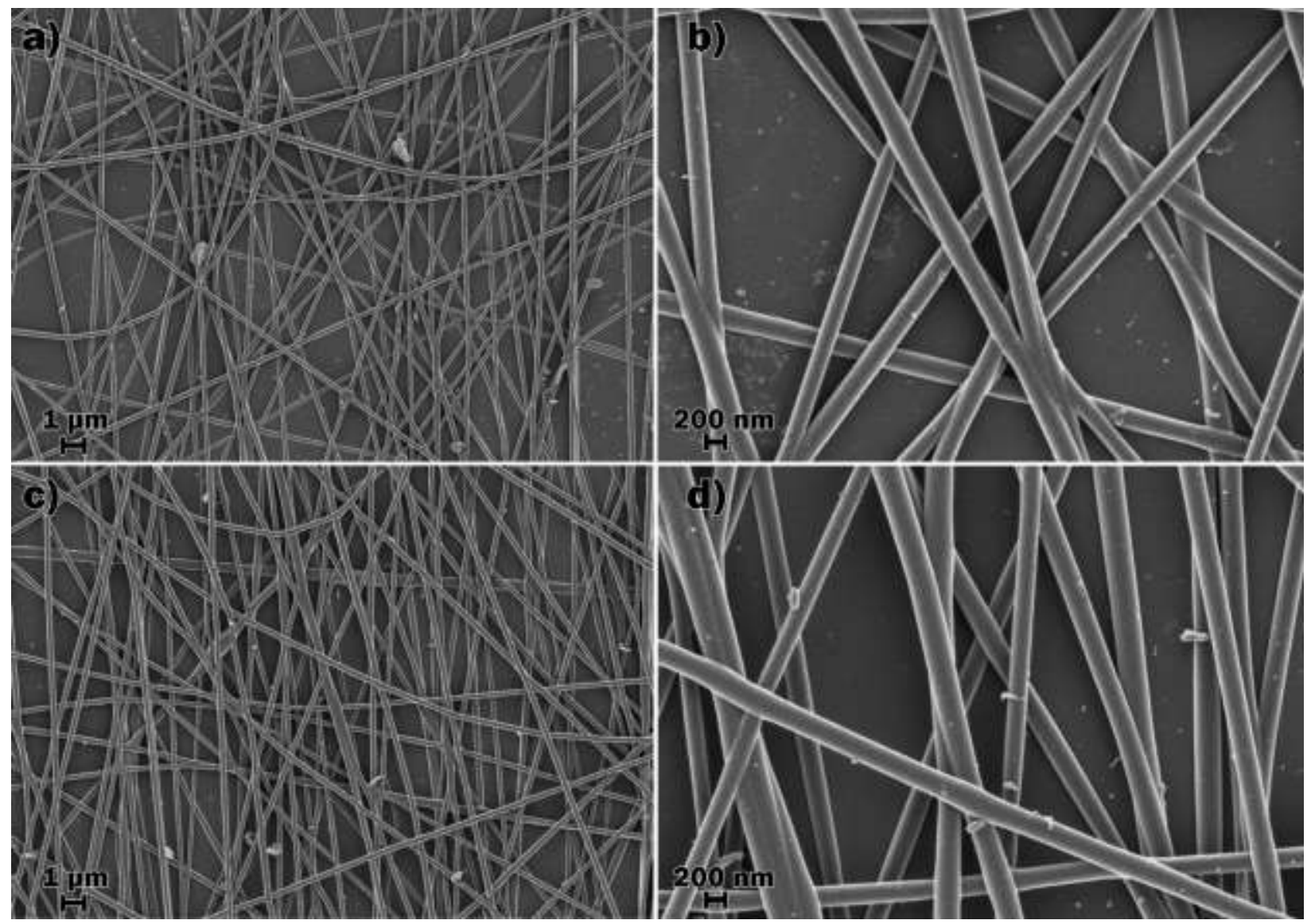

Figure 4 SEM micrographs of (a) and (b) PVA/graphene foam nano-fibers (c) and (d) PVA/ EG nanofibers at $1 \mathrm{kV}$ operating voltage and shown at different magnifications respectively. 
Figure 4 (a) - (d) show SEM images of PVA/graphene derived from GF (a and b) and EG ( $c$ and d) nanofibers at operating voltage of $1 \mathrm{kV}$ at different magnifications respectively. The morphology of the fibers produced using different concentrations of GF and EG is similar in diameter and density. In other words continuous nanofibers without beads or droplets are observed. In order to inspect if the graphene foam indeed filled the hollow PVA nanofibers, high magnification SEM of the fibers was taken at high voltage of $2 \mathrm{kV}$

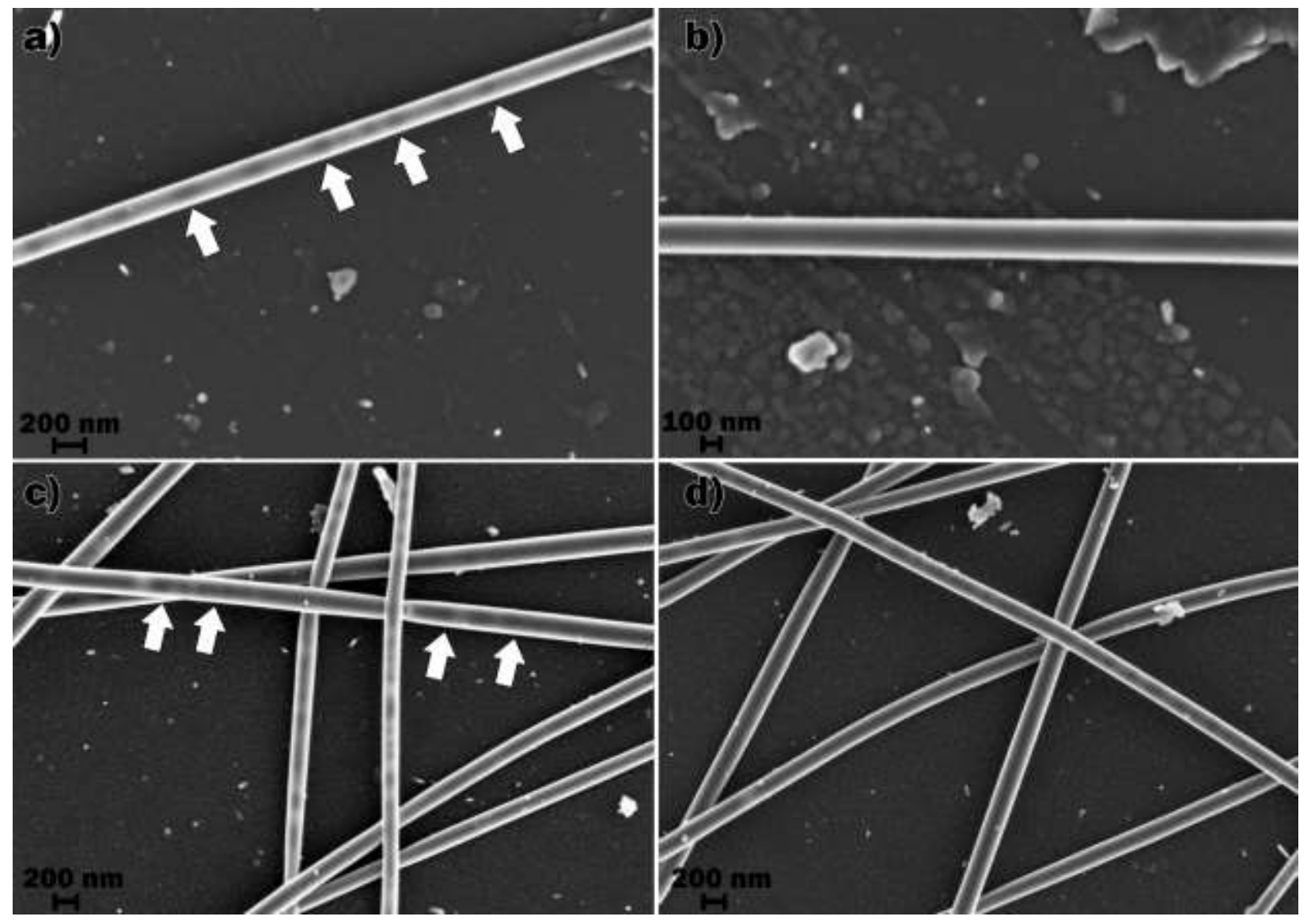

Figure 5 SEM micrographs of PVA/(GF and EG) nanofibers at $2 \mathrm{kV}$ operating voltage for (a) solution with $0.02 \mathrm{~g}$ GF concentration (b) solution with $0.08 \mathrm{~g}$ GF concentration (c) solution with $0.02 \mathrm{~g}$ EG concentration (d) solution with $0.08 \mathrm{~g} \mathrm{EG}$

For both nanofillers, namely GF and graphene derived from EG are shown in figure 5 (a) and (b) and figure 5 (c) and (d) for two different concentrations respectively. Figure 5 (a) and (c) show two different contrast of the fibers with outer side of the fiber being lighter and the inside consisting of dark dots as indicated by the arrows both for GF and 
EG fillers respectively. It is also observed that these dark dots seem to connect to form continuous structure in the fiber as the concentration of graphene is increased in the PVA solution (see figures 5 (b) and (d)), which adds to the evidence that the aligned dark dots correspond to graphene flakes in the PVA nanofibers.

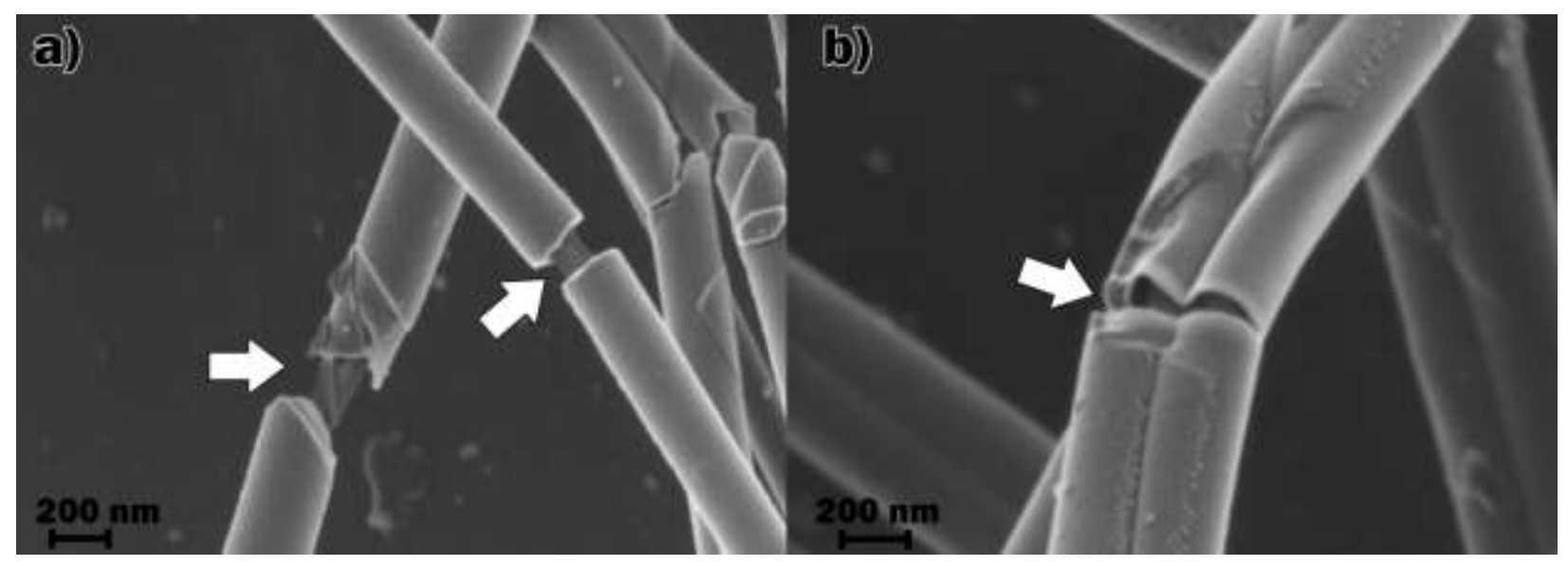

Figure 6 SEM micrographs of broken PVA/graphene foam nanofibers (from solution with $0.08 \mathrm{~g} \mathrm{GF}$ concentration)

To further confirm the presence of the graphene filler in the fibers, some fibers were physically broken by carefully scratching the fibers by razor blade to reveal the graphene nanofillers inside the fibers and this is shown in figure 6 for GF foam nanofiller with the concentration of $0.08 \mathrm{~g}$ as an example. It clearly appears that the graphene nanofillers are inside the hollow structure. This figure finally clearly proved that the electrospun nanofibers of PVA solution make the hollow structure and graphene nanofillers can be easily filled in the nanotube like structure. In fact, this could be attributed to the adhesion of the graphene and PVA [35], the strong interfacial adhesion between graphene-matrix which is due to the homogeneous dispersion of graphene in the polymer matrix and the surface tension of the PVA could lead to encapsulation of the graphene materials inside the hollow structure of the polymer after electrospinning [36].

\subsection{Decomposition Temperature}

Figure 7 (a) and (b) show typical TGA thermograms for PVA and PVA/graphene composites with their corresponding first order derivatives (DTGA) of weight loss as a 
function of temperature. The samples were measured in the temperature ranging from $25^{\circ} \mathrm{C}$ to $1000{ }^{\circ} \mathrm{C}$ with a constant ramping rate of $10^{\circ} \mathrm{C} / \mathrm{min}$ in air.
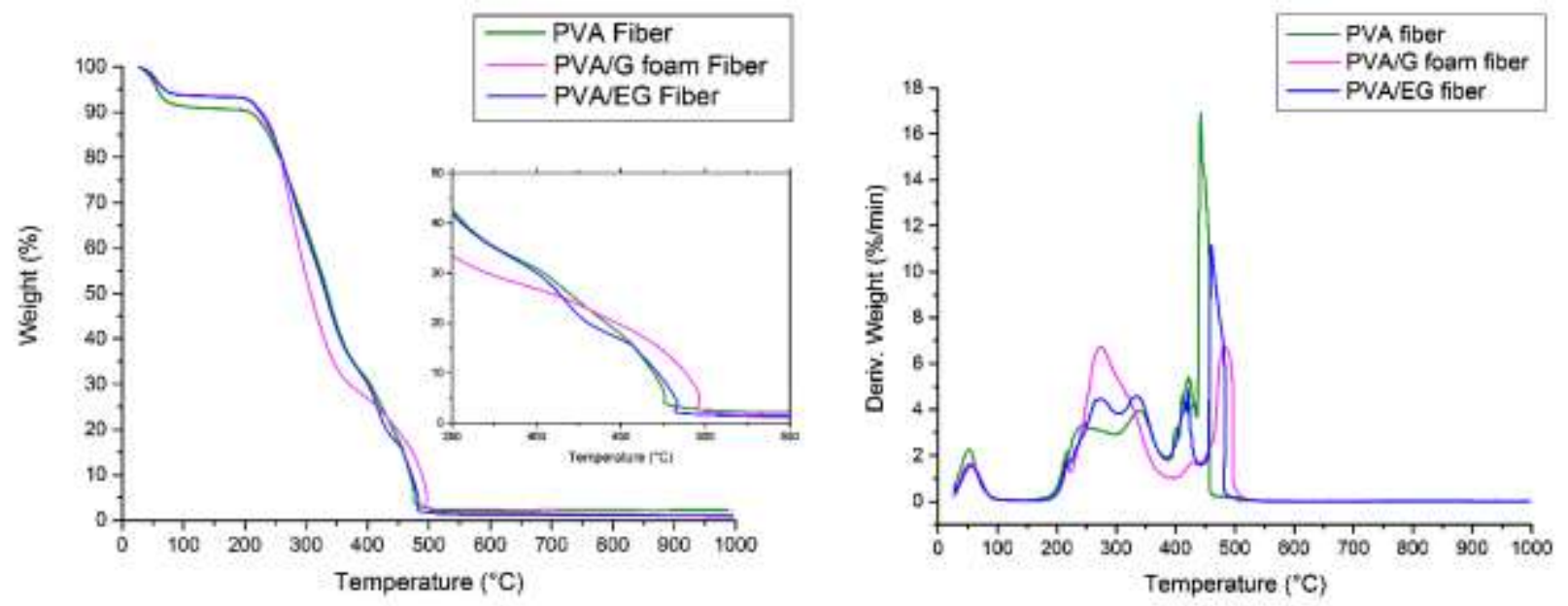

Figure 7 (a) TGA curves for electrospun PVA fibers with and without graphene derived from GF and EG and (b) The first order derivative of TGA for electrospun PVA fibers with and without graphene derived from GF and EG

Table 1 Degradation temperature of the baseline PVA and different graphene-loaded PVA nanofibers (from solution with $0.08 \mathrm{~g}$ GF and EG concentration)

\begin{tabular}{|c|c|c|c|}
\hline Samples & $\begin{array}{c}\text { First stage lose } \\
\text { weight }\end{array}$ & $\begin{array}{c}\text { Second stage } \\
\text { lose weight }\end{array}$ & $\begin{array}{c}\text { Third stage lose } \\
\text { weight }\end{array}$ \\
\hline PVA fiber & $25-145$ & $124-383$ & $383-460$ \\
\hline PVA/GF fiber & $25-143$ & $143-393$ & $393-542$ \\
\hline PVA/EG fiber & $25-141$ & $141-384$ & $384-559$ \\
\hline
\end{tabular}

TGA and DTGA show that all samples exhibit three distinct weight loss stages as depicted in table 1 above for the three different samples. The first range of $25-145^{\circ} \mathrm{C}$ corresponds to loss of moisture, physisorbed and chemisorbed water molecules [37], while the second one of $145-391^{\circ} \mathrm{C}$ corresponds to decomposition of the side chains of PVA (at this stage two peaks are observed with the first peak corresponding to the 
loss of $\mathrm{H}$ bond between PVA molecules and the second peak to the loss of $\mathrm{O}$ bond between $\mathrm{C}-\mathrm{O}$ ) and lastly $391-573{ }^{\circ} \mathrm{C}$ corresponding to the decomposition of the main chain of PVA[38,39].

The $2 \mathrm{D} \mathrm{sp}{ }^{2}$ sheet structure of graphene may act as a diffusion barrier, suppressing the decomposition of PVA which will result in increased decomposition temperature. Furthermore, the 2D sp ${ }^{2}$ sheet structure of graphene could help tightening the chain of PVA at higher temperatures. Above $600^{\circ} \mathrm{C}$, all TGA curves become flat which implies that mainly carbon residue are left. Evidently, the thermal decomposition of electrospun $\mathrm{PVA} /$ graphene composites fibers shift to the higher temperature range compared to electrospun PVA fibers. This implies that the incorporation of graphene in PVA for electrospun fibers enhanced the thermal stability of electrospun PVA nanofibers. This thermal reinforcement of electrospun PVA fibers by nano-carbon fillers is very important for polymer nanofibers in different technological applications such as those mentioned in the introduction section.

\subsection{Conclusions}

In this study the hollow PVA nanofibers were just produced through optimization of electrospinning parameters and incorporation graphene nanofillers was achieved in order to improve the thermal properties of the resulting continuous fibers by the relatively simple electrospinning technique. Two different source of graphene were used for this work and all showed improved thermal properties of the fibers after incorporation as nanofillers. Overall the improved thermal and morphological homogeneity could be attributed to a number of factors such as the strong electric field which makes the liquid jet faster, resulting in decrease in the average diameter of the produced fibers. Other parameters such as shorter needle tip/collector distance all have a contributing effect to the final diameter of the fiber. However, it was also observed that when this distance is increased, it takes a longer for the fibers to evaporate the solvent during spinning which resulted in lower electric field effect and thus increased the fibers diameter. Furthermore, the addition of graphene to the solution produces thinner electrospun fibers. This is due to the enhanced conductivity of the electrospun solution. These results show that fibers produced by this method are promising for use in electrodes, 
conductive wires, smart fabrics, and other applications which require conductive filler inside the polymer nanofibers.

\section{Acknowledgments}

This work is based on research supported by the South African Research Chairs Initiative (SARChi) of the South African Department of Science and Technology (DST) and the National Research Foundation (NRF). Any opinion, findings and conclusions or recommendations expressed in this work are those of the authors and the NRF and DST do not accept any liability with regard thereto. B.F acknowledges the financial support from the University of Pretoria and the NRF Doctorate Innovation Fund for his study.

\section{References}

[1] S. Ramakrishna, An Introduction to Electrospinning and Nanofibers, World Scientific, 2005.

[2] S. Das, A.S. Wajid, S.K. Bhattacharia, M.D. Wilting, I. V. Rivero, M.J. Green, Electrospinning of polymer nanofibers loaded with noncovalently functionalized graphene, J. Appl. Polym. Sci. 128 (2013) 4040-4046. doi:10.1002/app.38694.

[3] M. Chowdhury, G. Stylios, Effect of experimental parameters on the morphology of electrospun Nylon 6 fibres, Int. J. Basic .... 10 (2010) 116-131.

[4] G.-M. Kim, Nanofibers, InTech, 2010. doi:10.5772/45660.

[5] A.S. Nain, J.C. Wong, C. Amon, M. Sitti, Drawing suspended polymer micro/nanofibers using glass micropipettes, Appl. Phys. Lett. 89 (2006) 183105. doi:10.1063/1.2372694.

[6] D. Almecija, D. Blond, J.E. Sader, J.N. Coleman, J.J. Boland, Mechanical properties of individual electrospun polymer-nanotube composite nanofibers, Carbon N. Y. 47 (2009) 2253-2258. doi:10.1016/j.carbon.2009.04.022.

[7] J.S. Jeong, J.S. Moon, S.Y. Jeon, J.H. Park, P.S. Alegaonkar, J.B. Yoo, Mechanical properties of electrospun PVA/MWNTs composite nanofibers, Thin Solid Films. 515 (2007) 5136-5141. doi:10.1016/j.tsf.2006.10.058. 
[8] C. Wang, Y. Li, G. Ding, X. Xie, M. Jiang, Preparation and characterization of graphene oxide/poly(vinyl alcohol) composite nanofibers via electrospinning, J. Appl. Polym. Sci. 127 (2013) 3026-3032. doi:10.1002/app.37656.

[9] X.-M. Sui, S. Giordani, M. Prato, H.D. Wagner, Effect of carbon nanotube surface modification on dispersion and structural properties of electrospun fibers, Appl. Phys. Lett. 95 (2009) 233113. doi:10.1063/1.3272012.

[10] S. Costa, E. Borowiak-Palen, M. Kruszyńska, A. Bachmatiuk, R.J. Kaleńczuk, Characterization of carbon nanotubes by Raman spectroscopy., Mater. Sci. 26 (2008) 433-441.

[11] T.W. Ebbesen, Carbon Nanotubes, Phys. Today. (1996) 26-32.

[12] S. Sahoo, S. Dhibar, G. Hatui, P. Bhattacharya, C.K. Das, Graphene/polypyrrole nanofiber nanocomposite as electrode material for electrochemical supercapacitor, Polymer (Guildf). 54 (2013) 1033-1042. doi:10.1016/j.polymer.2012.12.042.

[13] M.I. Katsnelson, Graphene: carbon in two dimensions, Mater. Today. 10 (2007) 20-27. doi:10.1016/S1369-7021(06)71788-6.

[14] C.D.S. Huh, K. Jung, Polypyrrole/graphene oxide composites with improved conductivity and solubility, 4spepro.org. 50 (2012) 10-11. doi:0.1021/ja034894.

[15] Y. Xu, W. Hong, H. Bai, C. Li, G. Shi, Strong and ductile poly(vinyl alcohol)/graphene oxide composite films with a layered structure, Carbon N. Y. 47 (2009) 3538-3543. doi:10.1016/j.carbon.2009.08.022.

[16] C. Soldano, A. Mahmood, E. Dujardin, Production, properties and potential of graphene, Carbon N. Y. 48 (2010) 2127-2150. doi:10.1016/j.carbon.2010.01.058.

[17] T. Peijs, R.J.M. van Vught, L.E. Govaert, Mechanical properties of poly(vinyl alcohol) fibres and composites, Composites. 26 (1995) 83-90. doi:10.1016/00104361(95)90407-Q.

[18] J. Wang, X. Wang, C. Xu, M. Zhang, X. Shang, Preparation of graphene/poly(vinyl alcohol) nanocomposites with enhanced mechanical properties and water resistance, Polym. Int. 60 (2011) 816-822. doi:10.1002/pi.3025.

[19] A. Anish Madhavan, S. Kalluri, D. K Chacko, T.A. Arun, S. Nagarajan, K.R. V. Subramanian, et al., Electrical and optical properties of electrospun TiO2graphene composite nanofibers and its application as DSSC photo-anodes, RSC Adv. 2 (2012) 13032. doi:10.1039/c2ra22091a. 
[20] T. Ramanathan, A.A. Abdala, S. Stankovich, D.A. Dikin, M. Herrera-Alonso, R.D. Piner, et al., Functionalized graphene sheets for polymer nanocomposites., Nat. Nanotechnol. 3 (2008) 327-31. doi:10.1038/nnano.2008.96.

[21] J.T. McCann, D. Li, Y. Xia, Electrospinning of nanofibers with core-sheath, hollow, or porous structures, J. Mater. Chem. 15 (2005) 735. doi:10.1039/b415094e.

[22] L. Block, L. Schmeling, A. Couto, Effect of binders on 500mg metformin hydrochloride tablets produced by wet granulation, Rev. Ciências .... 30 (2009) $145-152$.

[23] G. Klimchitskaya, V. Mostepanenko, Van der Waals and Casimir interactions between two graphene sheets, Phys. Rev. B. 87 (2013) 075439.

doi:10.1103/PhysRevB.87.075439.

[24] A. Bello, K. Makgopa, M. Fabiane, D. Dodoo-Ahrin, K.I. Ozoemena, N. Manyala, Chemical adsorption of $\mathrm{NiO}$ nanostructures on nickel foam-graphene for supercapacitor applications, J. Mater. Sci. 48 (2013) 6707-6712. doi:10.1007/s10853-013-7471-x.

[25] A. Bello, O.O. Fashedemi, J.N. Lekitima, M. Fabiane, D. Dodoo-Arhin, K.I. Ozoemena, et al., High-performance symmetric electrochemical capacitor based on graphene foam and nanostructured manganese oxide, AIP Adv. 3 (2013) 082118. doi:10.1063/1.4819270.

[26] A.C. Ferrari, Raman spectroscopy of graphene and graphite: Disorder, electronphonon coupling, doping and nonadiabatic effects, Solid State Commun. 143 (2007) 47-57. doi:10.1016/j.ssc.2007.03.052.

[27] L.M. Malard, M.A. Pimenta, G. Dresselhaus, M.S. Dresselhaus, Raman spectroscopy in graphene, Phys. Rep. 473 (2009) 51-87. doi:10.1016/j.physrep.2009.02.003.

[28] Q. Li, H. Chou, J.-H. Zhong, J.-Y. Liu, A. Dolocan, J. Zhang, et al., Growth of adlayer graphene on Cu studied by carbon isotope labeling., Nano Lett. 13 (2013) 486-90. doi:10.1021/nl303879k.

[29] P.S. Thomas, B.H. Stuart, A Fourier transform Raman spectroscopy study of water sorption by poly(vinyl alcohol), Spectrochim. Acta Part A Mol. Biomol. Spectrosc. 53 (1997) 2275-2278. doi:10.1016/S1386-1425(97)00167-4.

[30] V. Beachley, X. Wen, Effect of electrospinning parameters on the nanofiber diameter and length, Mater. Sci. Eng. C. 29 (2009) 663-668. doi:10.1016/j.msec.2008.10.037.Effect. 
[31] Q.P. Pham, U. Sharma, A.G. Mikos, Electrospinning of polymeric nanofibers for tissue engineering applications: a review., Tissue Eng. 12 (2006) 1197-211. doi:10.1089/ten.2006.12.1197.

[32] Y. Srivastava, I. Loscertales, M. Marquez, T. Thorsen, Electrospinning of hollow and core/sheath nanofibers using a microfluidic manifold, Microfluid. Nanofluidics. 4 (2007) 245-250. doi:10.1007/s10404-007-0177-0.

[33] D.H. Reneker, I. Chun, Nanometre diameter fibres of polymer, produced by electrospinning, Nanotechnology. 7 (1996) 216-223. doi:10.1088/0957$4484 / 7 / 3 / 009$

[34] N.M. Thoppey, R.E. Gorga, J.R. Bochinski, L.I. Clarke, Effect of Solution Parameters on Spontaneous Jet Formation and Throughput in Edge Electrospinning from a Fluid-Filled Bowl, Macromolecules. 45 (2012) 6527-6537. doi:10.1021/ma301207t.

[35] U. Khan, P. May, H. Porwal, K. Nawaz, J.N. Coleman, Improved adhesive strength and toughness of polyvinyl acetate glue on addition of small quantities of graphene., ACS Appl. Mater. Interfaces. 5 (2013) 1423-8. doi:10.1021/am302864f.

[36] W. Zheng, B. Shen, Wentao Zhai, Surface Functionalization of Graphene with Polymers for Enhanced Properties, in: Intech, 2013: pp. 207-234.

[37] R. Anbarasan, R. Pandiarajaguru, R. Prabhu, V. Dhanalakshmi, A. Jayalakshmi, B. Dhanalakshmi, et al., Synthesis, characterizations, and mechanical properties of structurally modified poly(vinyl alcohol), J. Appl. Polym. Sci. 117 (2010) 20592068. doi:10.1002/app.32033.

[38] X.M. Sui, C.L. Shao, Y.C. Liu, White-light emission of polyvinyl alcoholZnO hybrid nanofibers prepared by electrospinning, Appl. Phys. Lett. 87 (2005) 113115. doi:10.1063/1.2048808.

[39] A. Kumar, Fabrication of Bio-nanocomposite Nanofibers Mimicking the Mineralized Hard Tissues via Electrospinning Process, 2010. 